\title{
EFEITO DA APLICAÇÃO PRÉ-COLHEITA DE CÁLCIO NA QUALIDADE E NO TEOR DE NUTRIENTES DE MANGA 'TOMMY ATKINS'1
}

\author{
DAVI JOSÉ SILVA², MOHAMMAD MENHAZUDDIN CHOUDHURY², ALESSANDRA MONTEIRO SALVIANO MENDES², \\ BÁRBARA FRANÇADANTAS ${ }^{2}$
}

RESUMO - Com o objetivo de avaliar o efeito da aplicação pré-colheita de cálcio na qualidade e no teor de nutrientes de frutos de manga Tommy Atkins, foi realizado um experimento em um pomar comercial com dez anos de idade, localizado no município de Petrolina-PE. Foram avaliados sete tratamentos, sendo duas fontes comerciais de cálcio e três dosagens de cada fonte $(5,8 ; 11,6$ e 17,4 mmol L-1 de Ca na forma de Ca-quelatizado e 45,0; 90,0 e 135,0 mmol L-1 de Ca na forma de $\mathrm{CaCl}_{2} \cdot 2 \mathrm{H}_{2} \mathrm{O}$ ), além de um tratamento-controle. $\mathrm{O}$ delineamento experimental foi em blocos ao acaso, com quatro repetições. As aplicações dos tratamentos foram realizadas quinzenalmente, iniciadas quando os frutos apresentavam tamanho chumbinho (5 a $10 \mathrm{~mm})$ e estenderam-se até duas semanas antes da colheita. Foram realizadas seis pulverizações de uma calda contendo os tratamentos, sendo que, em cada aplicação, foram fornecidos 12,5 L/planta de calda. Os frutos foram colhidos no estádio de maturação 2. Quarenta frutos, agrupados em lotes de dez, foram acondicionados em caixas de papelão com capacidade para $6 \mathrm{~kg}$ e submetidos ao armazenamento por 0;20;30 e 40 dias sob refrigeração $\left(10.5 \pm 1.0^{\circ} \mathrm{C}\right.$ e $90 \pm 5 \%$ de UR). Depois de retirados da câmara fria, os frutos foram mantidos por cinco dias em sala de amadurecimento a $21 \pm 1{ }^{\circ} \mathrm{C}$ e $60 \pm 5 \%$ de UR. Foram determinados os teores de N, K, Ca e Mg na casca e na polpa dos frutos no lote equivalente ao tempo inicial de armazenamento e avaliada a incidência de colapso interno em todos os lotes. A aplicação de cálcio tanto na forma quelatizada quanto de sal solúvel aumentou as concentrações do nutriente na casca dos frutos. Aplicações de Caquelatizado mostraram-se eficientes em aumentar as concentrações de cálcio na polpa dos frutos, podendo contribuir para prevenir a ocorrência de colapso interno em curto período de armazenamento.

Termos para indexação: Mangifera indica, nutrição mineral, desordens fisiológicas, colapso interno.

\section{QUALITY AND NUTRIENT LEVEL OF MANGO CV. 'TOMMY ATKINS' AS AFFECTED BY CALCIUM APPLICATION BEFORE HARVEST}

\begin{abstract}
The effect of calcium on quality and nutrient level of mango cv. "Tommy Atkins" was studied when applied prior to harvest on a ten-year old orchard, in Petrolina, State of Pernambuco, Brazil. Three levels of two commercial calcium sources (5.8; 11.6 and $17.3 \mathrm{mmol} \mathrm{L}^{-1}$ of $\mathrm{Ca}$ as chelate $\mathrm{Ca}$ and $45.0 ; 90.0$ and $135.0 \mathrm{mmol} \mathrm{L}^{-1}$ of $\mathrm{Ca}$ as the soluble salt $\mathrm{CaCl}_{2} \cdot 2 \mathrm{H}_{2} \mathrm{O}$ ), and a control treatment were evaluated, in a randomized complete block design with four replications. Calcium was applied every two weeks, starting at the fruit set stage (fruits 5 to $10 \mathrm{~mm}$ long) up to two weeks before harvest. Six sprayings of solutions containing the calcium treatments were applied providing $12.5 \mathrm{~L}$ of the solution/plant in each application. The fruits were harvested in the ripening stage 2 . Forty fruits were grouped in four lots of ten, placed into cardboard boxes with capacity for $6 \mathrm{~kg}$ and stored for $0,20,30$ and 40 days under refrigeration $\left(10.5 \pm 1.0^{\circ} \mathrm{C}\right.$ and $\left.90 \pm 5 \% \mathrm{RH}\right)$. After removed from the cold chamber, the fruits were kept for five days in a ripening room at $21 \pm 1{ }^{\circ} \mathrm{C}$ and $60 \pm 5 \% \mathrm{RH} . \mathrm{N}, \mathrm{K}, \mathrm{Ca}$ and $\mathrm{Mg}$ contents in the flesh and skin were determined in the lot equivalent to the shortest time of storage and the incidence of internal breakdown was evaluated in all four lots. Calcium application in both chelate and soluble salt forms increased the nutrient contents in the skin. Applications of chelate $\mathrm{Ca}$ showed to be efficient in increasing the calcium contents in the fruit flesh, contributing to prevent the occurrence of internal breakdown in short storage periods.
\end{abstract}

Index Terms: Mangifera indica, mineral nutrition, physiological disorders, internal breakdown.

\section{INTRODUÇÃO}

O colapso interno encontra-se no grupo de desordens fisiológicas da mangueira, conhecidas como "internal breakdown", atribuídas a desequilíbrios no metabolismo que tem causas diversas, geralmente relacionadas a fatores no ambiente pré e pós-colheita (Wainwright \& Burbage, 1989). Estas desordens fisológicas são classificadas de acordo com a sua sintomatologia em "soft-nose", "jelly seed", "stem-end-cavity," "spongy tissue" (Raymond et al., 1998; Evangelista, 1999).

Os fatores pré-colheita que podem predispor o fruto ao desenvolvimento de desordens, estão relacionados com a posição do fruto na árvore, as características do local de frutificação, a nutrição mineral e de carboidratos para o desenvolvimento do fruto, as relações hídricas e as respostas à temperatura. Por outro lado, os fatores pós-colheita são altas

'Trabalho 046-07). Recebido em : 28-02-2007. Aceito para publicação em: 06-09-2007. Trabalho realizado com recursos do convênio Embrapa/ PRODETAB

${ }_{2}^{2}$ Pesquisador, Embrapa Semi-Árido, BR 428, km 152, CEP 56302-970, Petrolina-PE, E-mail: davi@cpatsa.embrapa.br, mohammad@cpatsa.embrapa.br, amendes@cpatsa.embrapa.br, barbara@cpatsa.embrapa.br 
temperaturas e altos níveis de $\mathrm{CO}_{2}$ durante o armazenamento (Ferguson et al., 1999). Estes mesmos autores ainda relatam que a concentração de cálcio nos frutos, nutriente mais comumente associado com as desordens pós-colheita, depende desses fatores pré-colheita.

As concentrações de cálcio e nitrogênio parecem estar diretamente relacionadas com a incidência de colapso interno na mangueira. Cracknell et al. (2004) observaram que a incidência de colapso interno em manga 'Tommy Atkins' mostrou alta correlação positiva com o conteúdo de $\mathrm{N}$ e correlação negativa com o conteúdo de $\mathrm{Ca}$.

Em frutos da variedade Tommy Atkins, Assis et al. (2004) sugerem que as relações $\mathrm{N} / \mathrm{Ca}$ e $\mathrm{K} / \mathrm{Ca}$ são fatores importantes para a estabilidade das células da polpa dos frutos da mangueira. Estes autores também observaram que o magnésio parece estar relacionado ao problema, uma vez que os teores deste elemento foram significativamente maiores nos frutos sadios, ao passo que a relação $\mathrm{K} / \mathrm{Mg}$ também apresentou tendência de ser mais elevada nos frutos com sintomas de distúrbios fisiológicos.

Este trabalho teve como objetivo avaliar o efeito da aplicação pré-colheita de cálcio na qualidade e no teor de nutrientes em manga 'Tommy Atkins'.

\section{MATERIAL E MÉTODOS}

O experimento foi realizado em um pomar comercial, localizado no município de Petrolina-PE, tendo iniciado em novembro de 2004. Mangueiras da variedade Tommy Atkins, plantadas no espaçamento de $8,0 \times 5,0 \mathrm{~m}$, foram selecionadas em um pomar com 10 anos de idade. Foram avaliados sete tratamentos, sendo duas fontes comerciais de cálcio e três dosagens de cada fonte $\left(5,8 ; 11,6\right.$ e 17,4 $\mathrm{mmol} \mathrm{L}^{-1}$ de Ca na forma de Ca-quelatizado e 45,0; 90,0 e 135,0 $\mathrm{mmol} \mathrm{L}^{-1}$ de Ca na forma de $\mathrm{CaCl}_{2} \cdot 2 \mathrm{H}_{2} \mathrm{O}$ ), além de um tratamento-controle (testemunha). Foram realizadas seis pulverizações foliares de uma calda contendo os tratamentos, sendo que, em cada aplicação, foram fornecidos 12,5 litros por planta de calda. As pulverizações foliares iniciaram-se quando os frutos alcançaram o tamanho "chumbinho" (5 a $10 \mathrm{~mm}$ ) e foram realizadas quinzenalmente até duas semanas antes da colheita. $\mathrm{O}$ delineamento experimental foi de blocos ao acaso, com sete tratamentos e quatro repetições. A unidade experimental foi composta por cinco plantas.

Os frutos foram colhidos no estádio de maturação 2, cuja polpa apresenta cor creme-amarela (Alves et al., 2002), e foram transportados para o Laboratório de Qualidade Mercadológica da Embrapa Semi-Árido, em Petrolina-PE, onde foi realizado o corte do pedúnculo, a lavagem e a secagem dos frutos ao ar. Em seguida, foram selecionados 40 frutos de cada unidade experimental, os quais foram agrupados em quatro lotes, contendo 10 frutos cada. Os lotes foram acondicionados em caixas de papelão com capacidade para $6 \mathrm{~kg}$. O primeiro lote foi utilizado para a realização das análises físicas e químicas, caracterizando a qualidade dos frutos no tempo inicial de armazenamento. Cada fruto constituiu uma repetição. Os demais foram submetidos ao armazenamento por 20; 30 e 40 dias sob refrigeração $\left(10,5 \pm 1{ }^{\circ} \mathrm{C}\right.$ e $90 \pm 5 \%$ de UR) para a avaliação dos atributos de qualidade (peso médio de frutos, teor de sólidos solúveis totais, acidez total titulável e incidência de colapso interno). Depois da retirada da câmara fria, os frutos foram mantidos por cinco dias na condição de vida de prateleira sob $21 \pm 1{ }^{\circ} \mathrm{C}$ e $60 \pm 5 \%$ de UR.

As análises químicas foram realizadas no Laboratório de Solo e Planta da Embrapa Semi-Árido, em Petrolina-PE. Os frutos foram separados em casca e polpa, secados em estufa a $65-70^{\circ} \mathrm{C}$, para a obtenção da matéria seca. Em seguida, as amostras foram submetidas à digestão nítrico-perclórica, para a determinação dos teores do Ca e $\mathrm{Mg}$ por espectrometria de absorção atômica e do K por fotometria de chama, e digestão sulfúrica, para a determinação do nitrogênio por destilação (Malavolta et al., 1997).

A freqüência de incidência de colapso interno, expressa em percentagem, foi estimada pela relação entre o número de frutos que apresentavam sintomas de colapso interno e o número de frutos sadios de cada lote. Frutos que apresentavam podridões generalizadas não foram considerados nesse cálculo.

Os dados obtidos foram submetidos à análise de variância após a verificação de sua aderência à distribuição normal, pelo teste de Kolmogorov-Smirnov (Castro Neto, 2002). As médias foram comparadas pelo teste Tukey, a $5 \%$ de probabilidade (Castro Neto, 2002).

\section{RESULTADOS E DISCUSSÃO}

A concentração de nutrientes em amostras de folhas coletadas antes da aplicação dos tratamentos (Tabela 1) revela que os teores de N, K, Ca e Mg estão dentro da faixa de teores adequados considerada por Silva et al. (2002), e que as plantas da área experimental apresentavam uniformidade, pois não há diferença estatística entre os tratamentos para os nutrientes em questão. Contudo, a relação N/Ca está acima de 0,5 para a maior parte dos tratamentos, o que deixa os frutos de variedades monoembriônicas, como 'Tommy Atkins', suscetíveis à manifestação de sintomas de desordens fisiológicas. O efeito da relação N/Ca nas folhas foi relatado por Young e Miner (1961) como indicador da incidência de "soft nose", que sugerem o valor 0,5 como limite superior de tal relação. Nas variedades 'Tommy Atkins' (Pinto et al., 1994) e 'Keitt' (Evangelista, 1999), a incidência de desordens foi relacionada a relações N/Ca elevadas nas folhas, devido a níveis excessivos de $\mathrm{N}$ e deficientes de $\mathrm{Ca}$.

A aplicação de cálcio tanto na forma quelatizada quanto de sal solúvel contribuiu para o aumento das concentrações do nutriente na casca da manga, embora não tenham existido diferenças significativas entre as duas formas de cálcio aplicadas (Tabela 2). Isto é corroborado pelas relações $\mathrm{N} / \mathrm{Ca}$, que apresentam valores inferiores a 4,6 nos tratamentos que receberam cálcio. Embora os tratamentos que receberam cálcio na forma quelatizada tenham apresentado teores de potássio aparentemente menores, não houve diferença entre estes e os demais tratamentos que receberam cálcio na forma de sal solúvel, quando se observam as relações $\mathrm{K} / \mathrm{Ca}$. A aplicação de uma pasta de vaselina contendo Ca-EDTA ao pedúnculo dos frutos de manga 'Sensation' causou redução dos teores de K na casca e no interior do pedúnculo tanto na área tratada quanto fora dela 
(Hermoso et al., 1997).

Os teores de cálcio encontrados na casca dos frutos de manga são maiores que aqueles da polpa (Tabelas 2 e 3 ). O maior teor de cálcio na casca dos frutos pode ser justificado pelo fato de que as células da polpa apresentam vacúolos maiores, organelas de reservas e grandes porções citoplasmáticas, onde se encontra apenas o cálcio ligado a enzimas, o que é mais compatível com as funções citoplasmáticas. Já na casca, os tecidos têm função de proteção, contendo células menores e proporcionalmente maior quantidade de parede celular, constituídas por fibras celulósicas ricas em pectatos de cálcio e magnésio (Gunjate et al., 1979).

$\mathrm{Na}$ polpa, aplicações de Ca-quelatizado mostraram-se eficientes em aumentar as concentrações de cálcio (Tabela 3). As relações $\mathrm{N} / \mathrm{Ca}$ e $\mathrm{K} / \mathrm{Ca}$ foram significativamente maiores nos tratamentos que receberam as duas menores doses de cálcio na forma solúvel. Segundo Assis et al. (2004), altas relações N/Ca e $\mathrm{K} / \mathrm{Ca}$ afetam a estabilidade das células da polpa dos frutos da mangueira, predispondo-os a desordens fisiológicas. Assim, a concentração de cálcio na polpa parece ter refletido melhor o efeito dos tratamentos, embora os autores supracitados considerem que a concentração de nutrientes da casca pode refletir melhor a condição da fisiopatia do que a concentração dos nutrientes determinada na polpa dos frutos.

O peso médio dos frutos oscilou entre 417,23 a $615,23 \mathrm{~g}$, não havendo diferença estatística entre os tratamentos avaliados (Tabela 4). Houve correlação $(0,43)$ significativa a $5 \%$ de probabilidade, entre o peso médio dos frutos e a incidência de colapso interno no lote referente ao segundo período de armazenamento de frutos ( $20+5$ dias $)$.

Não houve efeito dos tratamentos sobre o teor de sólidos solúveis totais (SS) nem sobre a acidez total titulável (AT), com aumento do primeiro e redução do segundo em função do aumento do tempo de armazenamento. Isso refletiu-se no aumento da relação SS/AT até $30+5$ dias de armazenamento dos frutos (Tabela 5). Não houve aumento dos valores de SS nem da relação $\mathrm{SS} / \mathrm{AT}$ nos frutos que apresentaram maior incidência de colapso interno (Tabela 6). As correlações entre AT e a incidência de colapso interno foram de $-0,77$ e 0,82 para os tempos de $30+5$ e $20+5$ dias de armazenamento dos frutos, respectivamente, significativas a $5 \%$ de probabilidade. Normalmente, esses aumentos de SS e na relação SS/AT são conseqüência do próprio processo de maturação.

A incidência de colapso interno aumentou em função do tempo de armazenamento dos frutos, mas não houve diferença estatística entre os tratamentos em nenhum dos períodos de armazenamento (Tabela 6). Embora não tenham ocorrido diferenças significativas entre os tratamentos com relação à freqüência de incidência de colapso interno, houve maior incidência nos frutos que apresentaram as maiores relações N/ $\mathrm{Ca}$ e $\mathrm{K} / \mathrm{Ca}$ na polpa do lote correspondente ao segundo período de armazenamento de frutos. Houve correlação significativa entre a freqüência da incidência de colapso interno e as relações N/Ca $(r=0,52)$ e $N / K(r=0,53)$ na polpa, no segundo período de armazenamento de frutos. Na casca, as análises dos frutos do lote correspondente ao quarto período de armazenamento $(40+5$ dias) apresentam correlações significativas entre a freqüência da incidência de colapso interno e as relações $\mathrm{N} / \mathrm{Ca}(\mathrm{r}=0,78)$ e K/ $\mathrm{Ca}(\mathrm{r}=0,81)$ nos tratamentos que receberam cálcio quelatizado e $\mathrm{N} / \mathrm{Mg}(\mathrm{r}=0,73)$ e $\mathrm{Ca} / \mathrm{Mg}(\mathrm{r}=0,77)$ nos tratamentos que receberam cálcio na forma de sal solúvel. As relações observadas entre nitrogênio, potássio e cálcio podem ser explicadas pelo fato de que o nitrogênio e o potássio são absorvidos e distribuídos com rapidez nos órgãos e tecidos vegetais tanto pelo floema quanto pelo xilema (Mengel \& Kirkby, 1987). A absorção de cálcio, por sua vez, é bem menos eficiente que dos primeiros, podendo ser inibida pelo excesso de potássio. Além disso, os vasos do floema, maior provedor de nutrientes para os frutos, via de regra, contêm altas concentrações de potássio e concentrações de cálcio muito baixas.

A deficiência de cálcio nos frutos pode ser causada não somente pela baixa absorção, mas também pela competição entre os pontos de crescimento da planta e dos frutos pelo cálcio disponível. Níveis elevados de nitrogênio, os quais aumentam o crescimento vegetativo e também induzem a um desenvolvimento mais rápido do fruto, resultam em uma redução da concentração de cálcio no floema, aumentando o risco de que a concentração de cálcio nos tecidos do fruto seja reduzida para valores abaixo do nível crítico necessário à manutenção da integridade da membrana e estabilidade da parede celular (Marchner, 1995).

Dessa forma, a aplicação de cálcio quelatizado em baixa concentração $\left(5,8 \mathrm{mmol} \mathrm{L}^{-1}\right)$ mostrou-se eficiente em aumentar as concentrações de cálcio na polpa dos frutos, podendo contribuir para a prevenção de desordens fisiológicas em curto período de armazenamento. Hermoso et al. (1997) mostraram que aplicações de uma pasta de vaselina contendo Ca-EDTA ao pedúnculo de frutos de manga 'Sensation' reduziram significativamente a incidência de colapso interno, sendo mais eficientes que aplicações de soluções contendo $\mathrm{CaCl}_{2}, \mathrm{Ca}-\mathrm{EDTA}, \mathrm{KCl}$ ou $\mathrm{MgCl}_{2}$.

TABELA 1 - Teor foliar de nutrientes nas folhas de mangueira da área experimental antes da aplicação dos tratamentos. Petrolina-PE, 2004-2005.

\begin{tabular}{ccccc}
\hline Tratamento $^{1}$ & \multicolumn{4}{c}{ Nutriente $(\mathrm{g} / \mathrm{kg})$} \\
\cline { 2 - 5 } & $\mathrm{N}$ & $\mathrm{K}$ & $\mathrm{Ca}$ & $\mathrm{Mg}$ \\
\hline $5,8 \mathrm{CaQ}$ & 14,43 & 10,88 & 25,68 & 2,35 \\
$11,6 \mathrm{CaQ}$ & 13,50 & 11,63 & 24,73 & 2,28 \\
$17,4 \mathrm{CaQ}$ & 13,95 & 11,13 & 25,43 & 2,25 \\
$45,0 \mathrm{CaN}$ & 12,70 & 10,63 & 27,15 & 2,15 \\
$90,0 \mathrm{CaN}$ & 14,08 & 10,75 & 27,38 & 2,08 \\
$135,0 \mathrm{CaN}$ & 14,35 & 11,00 & 27,55 & 2,05 \\
Testemunha & 15,30 & 10,38 & 27,10 & 2,10 \\
\hline${ }^{1} \mathrm{CaQ} \cdot \mathrm{mmol} \mathrm{L}^{-1}$ de $\mathrm{Ca}$ & quelatizado; CaN: $\mathrm{mmol} \mathrm{L}^{-1}$ de Ca não-quelatizado.
\end{tabular}

${ }^{1} \mathrm{CaQ}$ : mmol L ${ }^{-1}$ de $\mathrm{Ca}$ quelatizado; $\mathrm{CaN}$ : $\mathrm{mmol} \mathrm{L}^{-1}$ de $\mathrm{Ca}$ não-quelatizado. 
TABELA 2 - Teores ( $\mathrm{g} / \mathrm{kg}$ ) e relações entre nutrientes na casca de manga 'Tommy Atkins' em função dos tratamentos aplicados em pré-colheita. PetrolinaPE, 2004-2005.

\begin{tabular}{cccccccccc}
\hline Tratamento $^{1}$ & $\mathrm{~N}$ & $\mathrm{~K}$ & $\mathrm{Ca}$ & $\mathrm{Mg}$ & $\mathrm{N} / \mathrm{K}$ & $\mathrm{N} / \mathrm{Ca}$ & $\mathrm{N} / \mathrm{Mg}$ & $\mathrm{K} / \mathrm{Ca}$ & $\mathrm{K} / \mathrm{Mg}$ \\
\hline $5,8 \mathrm{CaQ}$ & $8,89 \mathrm{ab}$ & $8,17 \mathrm{c}$ & $2,40 \mathrm{ab}$ & $1,62 \mathrm{~b}$ & $1,09 \mathrm{a}$ & $3,76 \mathrm{~b}$ & $5,50 \mathrm{a}$ & $3,46 \mathrm{~b}$ & $5,04 \mathrm{a}$ \\
$11,6 \mathrm{CaQ}$ & $8,60 \mathrm{~b}$ & $8,50 \mathrm{bc}$ & $2,20 \mathrm{ab}$ & $1,67 \mathrm{~b}$ & $1,01 \mathrm{ab}$ & $3,94 \mathrm{~b}$ & $5,23 \mathrm{a}$ & $3,91 \mathrm{~b}$ & $5,16 \mathrm{a}$ \\
$17,4 \mathrm{CaQ}$ & $8,70 \mathrm{~b}$ & $9,50 \mathrm{ab}$ & $2,19 \mathrm{ab}$ & $2,47 \mathrm{a}$ & $0,92 \mathrm{ab}$ & $4,00 \mathrm{~b}$ & $3,58 \mathrm{a}$ & $4,36 \mathrm{~b}$ & $3,90 \mathrm{a}$ \\
$45,0 \mathrm{CaN}$ & $8,70 \mathrm{~b}$ & $9,67 \mathrm{ab}$ & $2,02 \mathrm{~b}$ & $2,04 \mathrm{ab}$ & $0,91 \mathrm{~b}$ & $4,32 \mathrm{~b}$ & $4,33 \mathrm{a}$ & $4,83 \mathrm{~b}$ & $4,88 \mathrm{a}$ \\
$90,0 \mathrm{CaN}$ & $9,57 \mathrm{a}$ & $10,08 \mathrm{a}$ & $2,78 \mathrm{a}$ & $2,29 \mathrm{a}$ & $0,95 \mathrm{ab}$ & $3,44 \mathrm{~b}$ & $4,22 \mathrm{a}$ & $3,63 \mathrm{~b}$ & $4,44 \mathrm{a}$ \\
$135,0 \mathrm{CaN}$ & $9,28 \mathrm{ab}$ & $10,33 \mathrm{a}$ & $2,04 \mathrm{~b}$ & $2,34 \mathrm{a}$ & $0,90 \mathrm{~b}$ & $4,54 \mathrm{~b}$ & $4,03 \mathrm{a}$ & $5,09 \mathrm{~b}$ & $4,52 \mathrm{a}$ \\
Testemunha & $9,28 \mathrm{ab}$ & $10,00 \mathrm{a}$ & $1,35 \mathrm{c}$ & $2,21 \mathrm{ab}$ & $0,93 \mathrm{ab}$ & $7,13 \mathrm{a}$ & $4,30 \mathrm{a}$ & $7,62 \mathrm{a}$ & $4,62 \mathrm{a}$ \\
\hline $\mathrm{CV}(\%)$ & 5,41 & 4,85 & 10,89 & 10,01 & 9,01 & 30,23 & 19,32 & 31,86 & 16,59 \\
\hline
\end{tabular}

Médias seguidas da mesma letra nas colunas não diferem entre si, a $5 \%$ de probabilidade, pelo teste de Tukey.

${ }^{1} \mathrm{CaQ}$ : mmol L $\mathrm{L}^{-1}$ de $\mathrm{Ca}$ quelatizado; $\mathrm{CaN}$ : $\mathrm{mmol} \mathrm{L}^{-1}$ de $\mathrm{Ca}$ não-quelatizado.

TABELA 3 - Teores $(\mathrm{g} / \mathrm{kg})$ e relações entre nutrientes na polpa de manga 'Tommy Atkins' em função dos tratamentos aplicados em pré-colheita. PetrolinaPE, 2004-2005.

\begin{tabular}{cccccccccc}
\hline Tratamento $^{1}$ & $\mathrm{~N}$ & $\mathrm{~K}$ & $\mathrm{Ca}$ & $\mathrm{Mg}$ & $\mathrm{N} / \mathrm{K}$ & $\mathrm{N} / \mathrm{Ca}$ & $\mathrm{N} / \mathrm{Mg}$ & $\mathrm{K} / \mathrm{Ca}$ & $\mathrm{K} / \mathrm{Mg}$ \\
\hline $5,8 \mathrm{CaQ}$ & $7,64 \mathrm{a}$ & $10,33 \mathrm{~b}$ & $0,89 \mathrm{a}$ & $0,54 \mathrm{a}$ & $0,74 \mathrm{a}$ & $10,97 \mathrm{~b}$ & $14,16 \mathrm{a}$ & $15,19 \mathrm{~b}$ & $19,20 \mathrm{a}$ \\
$11,6 \mathrm{CaQ}$ & $7,44 \mathrm{a}$ & $10,83 \mathrm{~b}$ & $0,82 \mathrm{ab}$ & $0,76 \mathrm{a}$ & $0,69 \mathrm{a}$ & $9,91 \mathrm{~b}$ & $9,78 \mathrm{a}$ & $14,72 \mathrm{~b}$ & $14,20 \mathrm{a}$ \\
$17,4 \mathrm{CaQ}$ & $8,22 \mathrm{a}$ & $13,00 \mathrm{a}$ & $0,67 \mathrm{ab}$ & $0,62 \mathrm{a}$ & $0,63 \mathrm{a}$ & $12,98 \mathrm{~b}$ & $14,36 \mathrm{a}$ & $20,32 \mathrm{~b}$ & $22,43 \mathrm{a}$ \\
$45,0 \mathrm{CaN}$ & $7,83 \mathrm{a}$ & $11,58 \mathrm{ab}$ & $0,13 \mathrm{~b}$ & $0,62 \mathrm{a}$ & $0,68 \mathrm{a}$ & $60,90 \mathrm{a}$ & $13,11 \mathrm{a}$ & $90,00 \mathrm{a}$ & $19,31 \mathrm{a}$ \\
$90,0 \mathrm{CaN}$ & $7,54 \mathrm{a}$ & $10,25 \mathrm{~b}$ & $0,14 \mathrm{~b}$ & $0,55 \mathrm{a}$ & $0,74 \mathrm{a}$ & $56,75 \mathrm{a}$ & $13,83 \mathrm{a}$ & $77,11 \mathrm{a}$ & $18,80 \mathrm{a}$ \\
$135,0 \mathrm{CaN}$ & $7,54 \mathrm{a}$ & $10,67 \mathrm{~b}$ & $0,40 \mathrm{ab}$ & $0,75 \mathrm{a}$ & $0,71 \mathrm{a}$ & $19,82 \mathrm{~b}$ & $10,95 \mathrm{a}$ & $28,42 \mathrm{~b}$ & $15,64 \mathrm{a}$ \\
Testemunha & $7,73 \mathrm{a}$ & $10,33 \mathrm{~b}$ & $0,31 \mathrm{ab}$ & $0,52 \mathrm{a}$ & $0,75 \mathrm{a}$ & $25,42 \mathrm{~b}$ & $14,90 \mathrm{a}$ & $33,70 \mathrm{~b}$ & $19,93 \mathrm{a}$ \\
\hline $\mathrm{CV}(\%)$ & 5,17 & 4,67 & 50,22 & 19,78 & 8,45 & 78,60 & 23,08 & 78,42 & 23,00 \\
\hline
\end{tabular}

Médias seguidas da mesma letra nas colunas não diferem entre si, a $5 \%$ de probabilidade, pelo teste de Tukey.

${ }^{1} \mathrm{CaQ}$ : mmol L-1 de Ca quelatizado; $\mathrm{CaN}$ : $\mathrm{mmol} \mathrm{L}^{-1}$ de Ca não-quelatizado.

TABELA 4 - Peso médio (g) de frutos de manga 'Tommy Atkins' em diferentes períodos de armazenamento póscolheita, em função dos tratamentos aplicados em pré-colheita. Petrolina-PE, 2004-2005.

\begin{tabular}{ccccc}
\hline \multirow{2}{*}{ Tratamento $^{1}$} & \multicolumn{4}{c}{ Período de Armazenamento (dias) } \\
\cline { 2 - 5 } & 0 & $\left(20+5^{2}\right)$ & $(30+5)$ & $(40+5)$ \\
\hline $5,8 \mathrm{CaQ}$ & $588,40 \mathrm{a}$ & $566,66 \mathrm{a}$ & $438,85 \mathrm{a}$ & $586,06 \mathrm{a}$ \\
$11,6 \mathrm{CaQ}$ & $579,76 \mathrm{a}$ & $573,72 \mathrm{a}$ & $468,59 \mathrm{a}$ & $577,53 \mathrm{a}$ \\
$17,4 \mathrm{CaQ}$ & $615,23 \mathrm{a}$ & $587,91 \mathrm{a}$ & $446,40 \mathrm{a}$ & $596,09 \mathrm{a}$ \\
$45,0 \mathrm{CaN}$ & $590,63 \mathrm{a}$ & $585,65 \mathrm{a}$ & $434,85 \mathrm{a}$ & $545,78 \mathrm{a}$ \\
$90,0 \mathrm{CaN}$ & $582,91 \mathrm{a}$ & $582,09 \mathrm{a}$ & $452,12 \mathrm{a}$ & $591,36 \mathrm{a}$ \\
$135,0 \mathrm{CaN}$ & $560,34 \mathrm{a}$ & $609,62 \mathrm{a}$ & $417,23 \mathrm{a}$ & $569,48 \mathrm{a}$ \\
Testemunha & $568,20 \mathrm{a}$ & $579,95 \mathrm{a}$ & $464,99 \mathrm{a}$ & $547,67 \mathrm{a}$ \\
\hline CV $(\%)$ & 5,47 & 6,34 & 8,26 & 6,72 \\
\hline
\end{tabular}

Médias seguidas da mesma letra nas colunas não diferem entre si a $5 \%$ de probabilidade, pelo teste de Tukey.

${ }^{1} \mathrm{CaQ}$ : mmol L-1 de Ca quelatizado; $\mathrm{CaN}$ : $\mathrm{mmol} \mathrm{L}^{-1}$ de $\mathrm{Ca}$ não-quelatizado; ${ }^{2}$ Cinco dias sob a temperatura de $21 \pm 1{ }^{\circ} \mathrm{C}$ e $60 \pm 5 \%$ de UR ao final do período de 20;30 ou 40 dias de armazenamento sob refrigeração de 10,5 $\pm 1^{\circ} \mathrm{C}$ e $90 \pm 5 \%$ de UR.
TABELA 5 - Relação SS/AT de frutos de manga 'Tommy Atkins' em diferentes períodos de armazenamento póscolheita, em função dos tratamentos aplicados em pré-colheita. Petrolina-PE, 2004-2005.

\begin{tabular}{ccccc}
\hline Tratamento $^{1}$ & \multicolumn{4}{c}{ Período de Armazenamento (dias) } \\
\cline { 2 - 5 } & 0 & $\left(20+5^{2}\right)$ & $(30+5)$ & $(40+5)$ \\
\hline $5,8 \mathrm{CaQ}$ & $7,07 \mathrm{a}$ & $24,85 \mathrm{a}$ & $51,86 \mathrm{a}$ & $41,85 \mathrm{a}$ \\
$11,6 \mathrm{CaQ}$ & $8,31 \mathrm{a}$ & $29,44 \mathrm{a}$ & $61,85 \mathrm{a}$ & $45,97 \mathrm{a}$ \\
$17,4 \mathrm{CaQ}$ & $7,31 \mathrm{a}$ & $31,57 \mathrm{a}$ & $56,89 \mathrm{a}$ & $39,35 \mathrm{a}$ \\
$45,0 \mathrm{CaN}$ & $7,27 \mathrm{a}$ & $32,66 \mathrm{a}$ & $46,97 \mathrm{a}$ & $39,30 \mathrm{a}$ \\
$90,0 \mathrm{CaN}$ & $7,44 \mathrm{a}$ & $30,26 \mathrm{a}$ & $60,08 \mathrm{a}$ & $49,17 \mathrm{a}$ \\
$135,0 \mathrm{CaN}$ & $7,47 \mathrm{a}$ & $26,31 \mathrm{a}$ & $48,15 \mathrm{a}$ & $45,23 \mathrm{a}$ \\
Testemunha & $7,33 \mathrm{a}$ & $27,47 \mathrm{a}$ & $49,91 \mathrm{a}$ & $50,89 \mathrm{a}$ \\
\hline $\mathrm{CV}(\%)$ & 12,33 & 11,17 & 25,08 & 11,94 \\
\hline
\end{tabular}

Médias seguidas da mesma letra nas colunas não diferem entre si, a $5 \%$ de probabilidade, pelo teste de Tukey.

${ }^{1} \mathrm{CaQ}$ : $\mathrm{mmol} \mathrm{L}^{-1}$ de $\mathrm{Ca}$ quelatizado; $\mathrm{CaN}$ : $\mathrm{mmol} \mathrm{L}^{-1}$ de $\mathrm{Ca}$ não-quelatizado; ${ }^{2} \mathrm{Cinco}$ dias sob a temperatura de $21 \pm 1{ }^{\circ} \mathrm{C}$ e $60 \pm 5 \%$ de UR ao final do período de 20;30 ou 40 dias de armazenamento sob refrigeração de 10,5 $\pm 1{ }^{\circ} \mathrm{C}$ e $90 \pm 5 \%$ de UR.

TABELA 6 - Freqüência de incidência (\%) de colapso interno em frutos de manga 'Tommy Atkins' em diferentes períodos de armazenamento pós-colheita, em função dos tratamentos aplicados em pré-colheita. Petrolina-PE, 2004-2005.

\begin{tabular}{crccc}
\hline Tratamento $^{1}$ & \multicolumn{4}{c}{ Período de Armazenamento (dias) } \\
\cline { 2 - 5 } & \multicolumn{1}{c}{0} & $\left(20+5^{2}\right)$ & $(30+5)$ & $(40+5)$ \\
\hline $5,8 \mathrm{CaQ}$ & $12,50 \mathrm{a}$ & $12,50 \mathrm{a}$ & $47,62 \mathrm{a}$ & $79,17 \mathrm{a}$ \\
$11,6 \mathrm{CaQ}$ & $8,33 \mathrm{a}$ & $29,17 \mathrm{a}$ & $73,81 \mathrm{a}$ & $83,33 \mathrm{a}$ \\
$17,4 \mathrm{CaQ}$ & $4,17 \mathrm{a}$ & $39,88 \mathrm{a}$ & $84,13 \mathrm{a}$ & $87,50 \mathrm{a}$ \\
$45,0 \mathrm{CaN}$ & $16,67 \mathrm{a}$ & $41,67 \mathrm{a}$ & $90,48 \mathrm{a}$ & $45,83 \mathrm{a}$ \\
$90,0 \mathrm{CaN}$ & $4,17 \mathrm{a}$ & $41,67 \mathrm{a}$ & $95,83 \mathrm{a}$ & $70,83 \mathrm{a}$ \\
$135,0 \mathrm{CaN}$ & $12,50 \mathrm{a}$ & $33,33 \mathrm{a}$ & $90,48 \mathrm{a}$ & $54,16 \mathrm{a}$ \\
Testemunha & $12,50 \mathrm{a}$ & $27,77 \mathrm{a}$ & $87,50 \mathrm{a}$ & $62,50 \mathrm{a}$ \\
\hline CV $(\%)$ & 46,68 & 32,40 & 20,16 & 22,54 \\
\hline
\end{tabular}

Médias seguidas da mesma letra nas colunas não diferem entre si, a $5 \%$ de probabilidade, pelo teste de Tukey.

${ }^{1} \mathrm{CaQ}$ : $\mathrm{mmol} \mathrm{L}^{-1}$ de $\mathrm{Ca}$ quelatizado; $\mathrm{CaN}$ : $\mathrm{mmol} \mathrm{L}^{-1}$ de $\mathrm{Ca}$ não-quelatizado; ${ }^{2}$ Cinco dias sob a temperatura de $21 \pm 1{ }^{\circ} \mathrm{C}$ e $60 \pm 5 \%$ de UR ao final do período de $20 ; 30$ ou 40 dias de armazenamento sob refrigeração de 10,5 $\pm 1^{\circ} \mathrm{C}$ e $90 \pm 5 \%$ de UR.

\section{CONCLUSÕES}

1- A aplicação de cálcio tanto na forma quelatizada quanto de sal solúvel aumentou as concentrações do nutriente na casca dos frutos de manga Tommy Atkins.

2- O Ca-quelatizado foi mais eficiente em aumentar as concentrações de cálcio na polpa dos frutos, podendo contribuir para prevenir a ocorrência de colapso interno em curto período de armazenamento.

\section{AGRADECIMENTOS}

Os autores agradecem à Timbaúba Agrícola S.A., pela cessão de suas áreas de produção para a realização do experimento e à Sustainable Agro Solutions S.A.-SAS pelo fornecimento do produto quelatizado. 


\section{REFERÊNCIAS}

ALVES, R. E.; FILGUEIRAS, H. A. C.; MENEZES, J. B.; ASSIS, J. S. de; LIMA, M. A. C. de; AMORIM, T. B. F.; MARTINS, A. G. Colheita e pós-colheita. In: GENU, P. J. de C.; PINTO, A. C. de Q.(Ed.). A cultura da mangueira. Brasília, Embrapa Informação Tecnológica, 2002. cap. 17, p. 381-405.

ASSIS, J. S. de; SILVA, D. J.; MORAES, P. L.D. de. Equilíbrio nutricional e distúrbios fisiológicos em manga Tommy Atkins. Revista Brasileira de Fruticultura, Jaboticabal, v. 26, n. 2, p. 326-329, 2004.

COSTANETO, P.L.O. Estatística. 2. ed. São Paulo: Edgard Blucher. 2002.266p.

CRACKNELL, A.; CID, M.C.; SOCORRO, A.R.; FERNÁNDEZ, D.; ROSELL, P.; GALÁN, V. Effects of nitrogen and calcium supply on the incidence of internal fruit breakdown in 'Tommy Atkins' mangoes (Mangifera indica L.) grown in a soilless system. Acta Horticulturae, Wageningen, v.645, p.387-393, 2004.

EVANGELISTA, R. M. Qualidade de mangas 'Tommy Atkins' armazenadas sob refrigeração e tratadas com cloreto de cálcio pré-colheita. 1992. 129f. Tese. (Doutorado em Ciências dos Alimentos) - Universidade Federal de Lavras, Lavras, 1999.

FERGUSON, I.; VOLZ, R.; WOOLF, A. Preharvest factors affecting physiological disorders of fruit. Postharvest Biology and Technology, Amsterdan, v. 15, p.255-262, 1999.

GUNJATE, R. T.; TARE, S. J.; RANGWALA, A. D.; LIMAYE, V. P. Effect of pre-harvest and post-harvest calcium treatments on calcium content and occurrence of spongy tissue in Alphonso mango fruits. Indian Journal of Horticulture, Bangalore, v.36, n.2, p.140-144, 1979.
HERMOSO J.M.; GUIRADO, E.; GÓMEZ, R.; CASTILLA, A.; VELASCO, R.; FARRÉ, J.M. Effects of nutrients and growth substances on internal breakdown of Sensation mango fruits. Acta Horticulturae, Wageningen, v.455, p.92-99, 1997.

MALAVOLTA, E.; VITTI, G.C.; OLIVEIRA, S.A. de Avaliação do estado nutricional das plantas: princípios e aplicações. 2.ed. rev. e atual. Piracicaba: POTAFOS, 1997.319 p.

MARCHNER, $H$. Mineral nutrition of higher plants. $2^{\text {nd }} \mathrm{ed}$ London: Academic Press, 1995. 889p.

MENGEL K, KIRKBY, E. A. Principles of plant nutrition $4^{\text {th }}$ ed. Berna: International Potash institue, 1987. 687p.

PINTO, A. C. Q.; RAMOS, V. H. V.; JUNQUEIRA, N. T. V.; LOBATO, E.; SOUZA, D. M. G. Relação $\mathrm{Ca} / \mathrm{N}$ nas folhas e seu efeito na produção e qualidade da manga Tommy Atkins sob condições de cerrado. In: CONGRESSO BRASILEIRO DE FRUTICULTURA.13., 1994, Salvador. Resumos... v. 2, p.763.

RAYMOND, L.; SCHAFFER, B.; BRECHT. J. K.; CRANE, J. H. Internal breakdown in mango fruit: symptomology and histology of jelly seed, soft nose and stem-end-cavity. Postharvest Biology and Technology, Amsterdan, v.13, p.59-70, 1998.

SILVA, D. J.; QUAGGIO, J. A.; PINTO, P. A. da C.; PINTO, A. C. de Q.; MAGALHÃES, A. F. de J. Nutrição e adubação. In: GENU, P. J. de C.; PINTO, A. C. de Q.(Ed.). A cultura da mangueira. Brasília: Embrapa Informação Tecnológica, 2002. cap. 10, p.191221

WAINWRIGHT, H.; BURBAGE, M. B. Physiological disorders in mango (Mangifera indica L.) fruit. Journal of Horticultural Science, Ashfad, v. 64, n.2, p.125-135, 1989.

YOUNG, T. W.; MINER, J. T. Relationship of nitrogen and calcium to "soft-nose" disorder in mango fruits. Proceedings of the American Society for Horticultural Science, Alexandria, v.78, p.201-208, 1961. 